\title{
Prognosis and Prognostic Factors of Patients with Emergent Cerclage: A Japanese Single-Center Study
}

\author{
Ami Kobayashi, Hironori Takahashi (D), Shigeki Matsubara $(D)$, Yosuke Baba $(D)$ \\ Shiho Nagayama, Manabu Ogoyama $\left(\mathbb{D}\right.$, Kenji Horie $\mathbb{D}^{D}$, Hirotada Suzuki, Rie Usui,

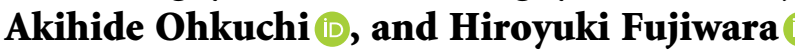

Department of Obstetrics and Gynecology, Jichi Medical University, 3311-1 Shimotsuke, Tochigi 329-0498, Japan

Correspondence should be addressed to Hironori Takahashi; hironori@jichi.ac.jp

Received 19 May 2021; Revised 11 October 2021; Accepted 14 December 2021; Published 26 December 2021

Academic Editor: Yoshitsugu Chigusa

Copyright $\odot 2021$ Ami Kobayashi et al. This is an open access article distributed under the Creative Commons Attribution License, which permits unrestricted use, distribution, and reproduction in any medium, provided the original work is properly cited.

\begin{abstract}
Objectives. The aims of this study were to clarify the following: (1) how often does prolonged pregnancy $\geq 34$ weeks occur in patients with emergent cerclage without progesterone and (2) the risk factors preventing such pregnancy continuation. Materials and Methods. This retrospective observational study was performed using medical records of patients for whom emergent cerclage had been performed between April 2006 and December 2018 in our institute. Results. Emergent cerclage was performed in 123 patients (median age: 34, interquartile range: 31-36). Primiparous patients numbered $44(36 \%)$ ). A history of spontaneous preterm birth (SPTB) was present in 30 (24\%). The median presurgical cervical length (CL) was $16(8-21) \mathrm{mm}$ at surgery. Of the 123,20 $(16 \%)$ were delivered at $33+6$ weeks or less ( $<34$ weeks). We conducted logistic regression analysis of the risk factors of SPTBs $<34$ weeks after cerclage. Three risk factors were identified that increased the risk of SPTB $<34$ weeks: presurgical CL $0 \mathrm{~mm}$ (odds ratio (OR): 5.30 ; 95\% confidence interval (CI): 1.58-17.7), a history of SPTB (OR: 4.65; 95\% CI: 1.38-15.7), and the presence of sludge (OR: 4.14; 95\% CI: 1.20-14.3). Conclusion. Three risk factors predicted SPTB <34 weeks after emergency cerclage without progesterone administration: unmeasurable CL $(\mathrm{CL} 0 \mathrm{~mm})$, a history of SPTB, and the presence of sludge on ultrasound. SPTB $<34$ weeks occurred after emergency cerclage in $16 \%$ of patients, being comparable with the recent data with progesterone.
\end{abstract}

\section{Introduction}

Treatment and prophylaxis for spontaneous preterm birth (SPTB) are challenging: administration of progesterone [1], tocolytic agents [2], antibiotics [3], and their combination $[4,5]$ have been reported to reduce SPTB incidence. Cervical cerclage has also been attempted in some patients. Cerclage is divided into two types: elective and emergent cerclage. The former is performed at 12-14 weeks' gestation for patients with historical indications. The latter, also referred to as rescue cerclage, is usually performed at 15-24 weeks for patients with an ultrasound-detectable shortened cervical length (CL) and patients with this latter cerclage, compared with those with the former clearly show poorer perinatal outcomes, especially PTB. Thus, patients after emergent cerclage require special attention, especially for PTB.
Progesterone has been widely recommended as a treatment for threatened PTB in global guidelines [6-8]. However, in Japan, progesterone is not yet a routine choice because national insurance does not cover it. Without progesterone, we Japanese obstetricians employ cerclage. The fundamental policy of our institute is: emergent cerclage should be performed for a patient with $\mathrm{CL}<25 \mathrm{~mm}$ at $<24$ weeks of gestation regardless of the presence or absence of a history of SPTB, if contraindications are absent (described later). Nonuse of progesterone gave us a chance to answer the following question: without progesterone, what perinatal outcomes will emergent cerclage yield? We attempted to answer two questions: (1) how often does prolonged pregnancy $\geq 34$ weeks occur (possible effectiveness of cerclage) and (2) what are the risk factors preventing such pregnancy continuation (risk factors of SPTB $<34$ weeks after cerclage). 


\section{Materials and Methods}

This study was approved by the ethics committee of our institute (20-070). We retrieved medical charts of patients for whom cervical cerclage was performed between April 2006 and December 2018 in our institute, in which approximately 1,000 high-risk deliveries are managed annually. Our treatment strategy for SPTB fundamentally adhered to the Japanese guidelines [9-11]. The delivery mode and timing were decided by attending obstetricians.

We performed emergent cerclage in patients with CL $<25 \mathrm{~mm}$ at less than 24 weeks of gestation irrespective of the presence or absence of a history of SPTB with patients' consent. Transvaginal ultrasound was routinely performed between 19 and 23 weeks of gestation. In high-risk SPTB patients, we measured CL weekly or biweekly from 15 to 24 weeks. Patients referred from other institutes due to shortened CL were also included. We included patients with shortened CL regardless of the presence or absence of preterm labor pains. If CL could not be measured because of cervical effacement and cervical shorting, we defined the condition as CL $0 \mathrm{~mm}$. We did not employ cerclage in the following patients (exclusion criteria): those with (1) active vaginal bleeding, (2) evident chorioamnionitis (e.g., white blood cell $\geq 15,000 / \mu \mathrm{L}$, high-grade fever, and fetal tachycardia), (3) prolapse of the membrane (bag) into the vagina, (4) rupture of the membrane, (5) continuous preterm labor pains despite administration of tocolytic agents, (6) lethal major fetal anomalies, and (7) those not consenting to the cerclage. We excluded iatrogenic preterm births including preeclampsia, medical complications, and non-reassuring fetal status before labor pains from this study. We also excluded placenta previa, fetal abnormalities, and multiple deliveries. Amniocentesis to measure subclinical infection was not performed. Although emergent cerclage was performed from 15 to 24 weeks' gestation, it was exceptionally performed at 24 to 26 weeks in a few cases based on patients' wishes and obstetricians' decisions.

Regarding the operative procedure, McDonald cerclage was performed as follows. After spinal anesthesia using ropivacaine or bupivacaine, patients were placed in a lithotomy position. If necessary, the Trendelenburg position was concomitantly employed. Using 1 monofilament nylon thread, we placed single McDonald cerclage as described as a standard technique. We included patients in whom the OS opened $1-4 \mathrm{~cm}$ and the membranes were visible: we pushed the membrane back into the uterus (membrane replacement) in some patients using a wet gauze and/or small balloon (Mini-Metro, Soft Medial, Tokyo, Japan). After the operation, systemic antibiotics (e.g., flomoxef sodium) were administered intravenously for more than 3 days. After the operation, the patients were hospitalized for more than one week. Ritodrine hydrochloride, magnesium sulfate, or their combination was administered as tocolysis in all patients. The attending obstetricians decided on the periods of these administrations and hospitalizations. Vaginal or intramuscular progesterone was not administered because it is not covered by national insurance. Vaginal ulinastatin, which is a granulocyte elastase inhibitor, was administered every day based on patients' wishes during hospitalization and also based on the Japanese guideline [9-11]. Blood analyses were performed more than once a week. The cerclage thread was removed at 36 weeks of gestation. When complete premature rupture of the membranes occurred, the thread was removed regardless of gestational weeks. We routinely performed transvaginal ultrasound once a week, using a 5-7.5 MHz transducer.

From electronic medical records, we retrieved maternal backgrounds (e.g., age, parity, mode of conception, smoking, presence of cervical polyp, a history of uterine surgery, a history of cesarean section (CS), and a history of SPTB), CL, presence/absence of sludge on ultrasound, vaginal bacterial culture, and the presence/absence of cervical elastase [12], gestational week at delivery, birth weight, fetal sex, and neonatal intensive care unit admission. Regarding the presence or absence of sludge, it was not familiar to general obstetricians until approximately 2011. Thus, we checked ultrasound images in medical charts in all the included patients. AK and HT checked and judged the presence or absence of sludge separately. If opinions were divided, AK judged it. The Mann-Whitney $U$-test and Fisher's exact test (two-tailed) were used to compare characteristics, maternal backgrounds, and outcomes between deliveries at less than 34 weeks' gestation $(+)$ vs. (-). Parameters significant $(P<0.15)$ on univariate analysis were used for logistic regression analysis. All analyses were performed using JMP Pro version 15 (SAS Institute, Tokyo, Japan), with $P<0.05$ considered significant.

\section{Results}

Table 1 shows patients' backgrounds. Cervical cerclage was performed in 338 patients during the study period. Of those, emergent cerclage was performed in 123 (median age: 34, interquartile range (IQR): 31-36). Primiparous patients numbered $44(36 \%)$. Pregnancies after assisted reproductive technology (ART) accounted for 13 (11\%). Histories of CS, myomectomy, cervical cerclage, and conization were noted in 21, 2, 7, and 2 patients, respectively. A history of SPTB was present in $30(24 \%)$. Slight bleeding at admission was noted in 7 (6\%). The median presurgical CL was 16 (IQR: 8-21) $\mathrm{mm}$. The presence of sludge in ultrasound was observed in 24 (16\%). Regarding vaginal culture, Lactobacillus and ureaplasma were present in $89(72 \%)$ and $42(34 \%)$, respectively. The prolapsed membrane was replaced by pushing-back at surgery in $22(18 \%)$. No uterine malformation was noted. Premature rupture of the membrane at surgery did not occur in any patients.

Table 2 shows the perinatal outcome of patients with emergent cerclage. Of the 123, $79(64 \%)$ and $44(36 \%)$ resulted in term deliveries and PTBs, respectively: of the latter 44, $20(16 \%)$ were delivered at $33+6$ weeks or less $(<34$ weeks). The median birth weight was $2,706 \mathrm{~g}$. NICU admission was required in 51 (41\%). Figure 1 shows a scatter plot of the 123 patients in both groups with or without a history of SPTB. All cases with both $\mathrm{CL}=0 \mathrm{~mm}$ and a history of SPTB resulted in SPTB $<34$ weeks. 
TABle 1: Patients' backgrounds.

\begin{tabular}{|c|c|}
\hline Characteristic & $n=123$ \\
\hline Age (years), median (IQR) & $34(31-36)$ \\
\hline$<30, n(\%)$ & $26(21)$ \\
\hline $30-34, n(\%)$ & $46(37)$ \\
\hline $35-39, n(\%)$ & $40(33)$ \\
\hline$\geq 40, n(\%)$ & $11(9)$ \\
\hline Primiparous, $n(\%)$ & $44(36)$ \\
\hline Pregnancy by ART, $n(\%)$ & $13(11)$ \\
\hline History of CS, $n(\%)$ & $21(17)$ \\
\hline History of myomectomy, $n$ (\%) & $2(2)$ \\
\hline History of cervical cerclage, $n(\%)$ & $7(6)$ \\
\hline History of conization, $n(\%)$ & $2(2)$ \\
\hline History of SPTB & $30(24)$ \\
\hline In the 2 nd trimester & $13(11)$ \\
\hline In the $3 \mathrm{rd}$ trimester & $17(14)$ \\
\hline Slight bleeding at admission, $n(\%)$ & $7(6)$ \\
\hline Presurgical CL (mm), median (IQR) & $16(8-21)$ \\
\hline Presurgical CL $<10 \mathrm{~mm}, n(\%)$ & $35(28)$ \\
\hline Presurgical CL $0 \mathrm{~mm}, n(\%)$ & $19(15)$ \\
\hline Presence of sludge in ultrasound, $n(\%)$ & $24(16)$ \\
\hline Cervical elastase positive, $n(\%)^{*}$ & $20(23)$ \\
\hline Presence of Lactobacillus, $n(\%)^{* *}$ & $89(72)$ \\
\hline Presence of ureaplasma, $n(\%)^{* * *}$ & $42(34)$ \\
\hline $\begin{array}{l}\text { Presurgical C-reactive protein }(\mathrm{mg} / \mathrm{dL}) \text {, median } \\
(\mathrm{IQR})\end{array}$ & $\begin{array}{c}0.21 \\
(0.10-0.37)\end{array}$ \\
\hline \multicolumn{2}{|l|}{ Procedure of cerclage } \\
\hline McDonald & $123(100)$ \\
\hline $\begin{array}{l}\text { Replacement of prolapsed membranes at surgery, } \\
n(\%)\end{array}$ & $22(18)$ \\
\hline
\end{tabular}

ART, assisted reproductive technology; CL, cervical length; IQR, interquartile range; SPTB, spontaneous preterm birth. ${ }^{*}$ Not examined in 37 patients, ${ }^{* *}$ not examined in 2 patients, ${ }^{* * *}$ not examined in 28 patients.

TABle 2: Perinatal outcome of patients with cervical cerclage.

\begin{tabular}{lc}
\hline & $n=123$ \\
\hline GA at delivery, $n(\%)$ & $20(16)$ \\
$33+6$ or less weeks & $24(20)$ \\
$34+0-36+6$ weeks & $79(64)$ \\
$\geq 37$ weeks & \\
GA at delivery with $33+6$ or & $4(3)$ \\
less weeks, $n$ (\%) & $6(5)$ \\
$<22$ weeks & $3(2)$ \\
$22+0201325+6$ weeks & $7(6)$ \\
$26+0-29+6$ weeks & $48(14-96)$ \\
$30+0-33+6$ weeks & $2706(2340-3030)$ \\
Duration between operation and & $7.44 \pm 1.74$ \\
discharge (days), median (IQR) & \\
Birth weight (grams), median $(\mathrm{IQR})$ & $8.64 \pm 1.01$ \\
Apgar score 1 mins, mean \pm SD & $51(41)$ \\
Apgar score 5 mins, mean \pm SD & \\
NICU admission, $n(\%)^{* *}$ &
\end{tabular}

GA, gestational age; IQR, interquartile range; NICU, neonatal intensive care unit; SD, standard deviation, * duration between operation and delivery, if delivery occurred during hospitalization. ${ }^{* *}$ Four abortive fetuses were excluded.

Table 3 shows a univariate analysis of risk factors of SPTB $<34$ weeks, obtained by the comparison between patients delivered before vs. after $<34$ weeks. The former ( $<34$ weeks), compared with the latter ( $\geq 34$ weeks), had (i) a significantly more frequent CL $0 \mathrm{~mm}(8(40 \%)$ vs. $12(12 \%)$, respectively; $(P=0.003)$ and (ii) significantly more frequent ultrasound-detectable sludge $(8(40 \%)$ vs. $16(16 \%)$, respectively $(P=0.029)$. Patients with SPTB $<34$ weeks frequently had a history of SPTB, although it was not significant (8 $(40 \%)$ vs. $22(21 \%)$, respectively $(P=0.091)$. The median duration between the cerclage and discharge showed a shorter tendency in patients with SPTB $<34$ weeks than those with $\geq 34$ weeks (35 (IQR: $21-84$ ) vs. 76 (IQR: 13-98) days, respectively $(P=0.057)$.

Table 4 shows logistic regression analysis of the risk factors of SPTBs <34 weeks after cerclage. Long-term tocolysis and hospitalization management were employed for the majority of patients in this study. Thus, the duration of hospitalization was not included in logistic regression analysis, although the $P$ value of the duration was 0.057 . Three risk factors were identified that increased the risk of SPTB $<34$ weeks: presurgical CL $0 \mathrm{~mm}$ (odds ratio (OR): 5.30; $95 \%$ confidence interval (CI): 1.58-17.7), a history of SPTB (OR: 4.65; 95\% CI: 1.38-15.7), and the presence of sludge (OR: 4.14; 95\% CI: 1.20-14.3).

\section{Discussion}

We made a few important observations in patients necessitating emergent cerclage without progesterone administration. SPTB $<34$ weeks occurred after emergency cerclage in $16 \%$ of patients. Three risk factors predicted SPTB $<34$ weeks: unmeasurable CL (CL $0 \mathrm{~mm}$ ), a history of SPTB, and the presence of sludge on ultrasound. These results may be similar to those of patients managed with progesterone administration.

A few studies have been reported regarding risk factors for SPTB after cerclage under different pre- or postoperative managements, including no routine tocolysis and progesterone administration. Fuchs et al. [13] reported that four risk factors, a history of PTB, cervical dilatation, membranes bulging into the vagina, and infection (white blood cell $\geq 13600 / \mathrm{mm}^{3}$ or CRP $>15 \mathrm{mg} / \mathrm{L}$ ), are associated with PTB $<32$ weeks. There are similarities and differences between Fuchs's study and our study. The similarities were that a history of PTB and cervical dilation (" $\mathrm{CL}=0 \mathrm{~mm}$ ") was associated with PTB. Contrarily, there were some differences in inclusion criteria and risk factors to be examined. $\mathrm{He}$ included patients with bulging bag into vagina, whereas we excluded them. Regarding infection, he took WBC and CRP into consideration; however, we evaluated sludge and cervical elastase. Another study concluded that, of patients receiving cerclage during 10-24 weeks, emergent cerclage, a history of conization, and CL $<25 \mathrm{~mm}$ were risk factors for PTB $<32$ weeks [14]. Inclusion criteria were also different from our study. The biggest difference was that patients with history-indicated cerclage were included in the previous study [14].

A history of SPTB was a significant risk for SPTB $<34$ weeks in this study. Numerous studies showed that a history of SPTB is a risk factor for SPTB. Of those, a systematic review and meta-analysis showed that the rate of SPTB $(<37$ weeks) was high as $30 \%$ in 55,197 patients with a history of a 


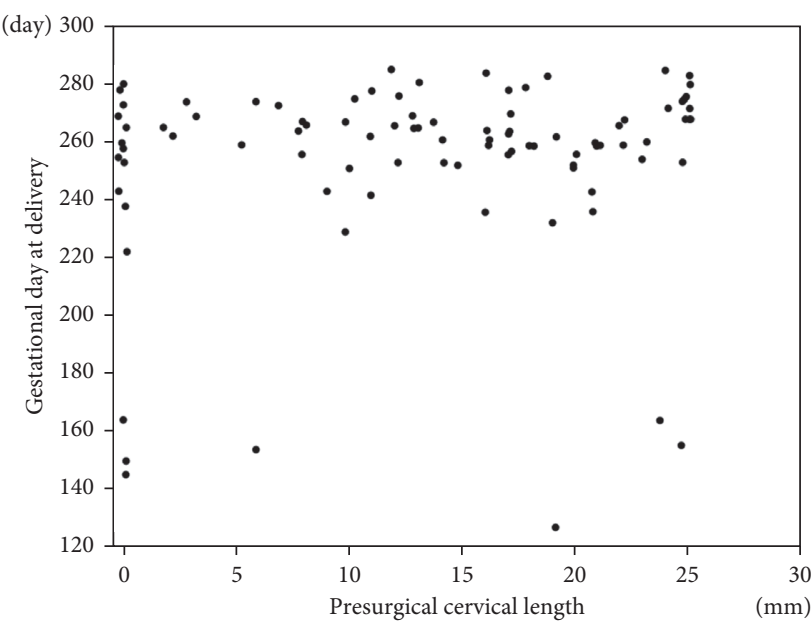

(a)

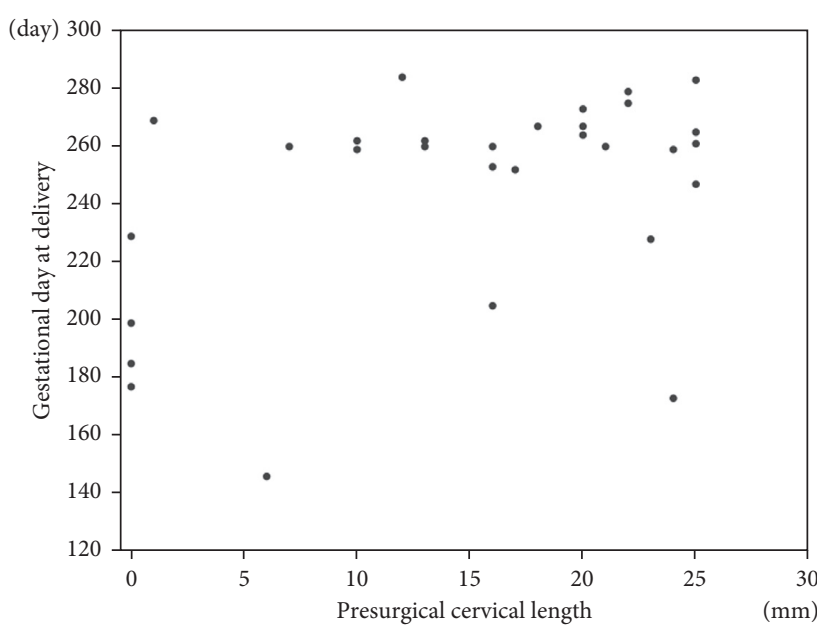

(b)

FIGURE 1: Scatter plot of included patients (123 patients) in both groups without (a) (93 patients) and with (b) (30 patients) a history of SPTB. $x$-axis: presurgical cervical length. $y$-axis: gestational age at delivery. 280 days $=40+0$ weeks of gestation. 259 days $=37+0$ weeks of gestation. 238 days $=34+0$ weeks of gestation. 210 days $=30+0$ weeks of gestation. 168 days $=24+0$ weeks of gestation. All cases with both $\mathrm{CL}=0 \mathrm{~mm}$ and a history of SPTB resulted in SPTB $<34$ weeks.

TABLE 3: Univariate analysis of risk factors among preterm deliveries less than 34 weeks.

\begin{tabular}{|c|c|c|c|}
\hline & $<34$ weeks $(n=20)$ & $\geq 34$ weeks $(n=103)$ & $P$-value \\
\hline Age, median (IQR) & $34(32-36)$ & $33(30-37)$ & 0.488 \\
\hline Primiparous, $n(\%)$ & $9(45)$ & $35(34)$ & 0.445 \\
\hline Pregnancy by ART, $n(\%)$ & $1(5)$ & $13(13)$ & 0.691 \\
\hline Presence of cervical polyp, $n(\%)$ & $1(5)$ & $7(7)$ & 1.000 \\
\hline History of cerclage, $n(\%)$ & $0(0)$ & $7(7)$ & 0.597 \\
\hline History of CS, $n(\%)$ & $1(5)$ & $20(19)$ & 0.192 \\
\hline History of myomectomy, $n(\%)$ & $1(5)$ & $1(1)$ & 0.300 \\
\hline Slight bleeding at admission, $n(\%)$ & $2(10)$ & $5(5)$ & 0.318 \\
\hline Presurgical CL, mm (IQR) & $8(0-21)$ & $16(10-21)$ & 0.082 \\
\hline Presurgical CL $0 \mathrm{~mm}, n(\%)$ & $8(40)$ & $12(12)$ & 0.003 \\
\hline Presence of sludge in ultrasound, $n(\%)$ & $8(40)$ & $16(16)$ & 0.029 \\
\hline Presence of Lactobacillus & $14(70)$ & $75(73)$ & 0.782 \\
\hline Presence of ureaplasma & $7(35)$ & $35(34)$ & 1.000 \\
\hline Cervical elastase positive, $n(\%)$ & $4(20)$ & $16(16)$ & 0.272 \\
\hline GA at emergency cerclage, week (IQR) & $21(18-24)$ & $22(20-23)$ & 0.377 \\
\hline History of SPTB, $n(\%)$ & $8(40)$ & $22(21)$ & 0.091 \\
\hline History of cerclage, $n(\%)$ & $1(5)$ & $9(9)$ & 0.687 \\
\hline Duration between cerclage and discharge (days), median (IQR)* & $35(21-84)$ & $76(13-98)$ & 0.057 \\
\hline
\end{tabular}

ART, assisted reproductive technology; CL, cervical length; CS, cesarean section; GA, gestational age; IQR, interquartile range; SPTB, spontaneous preterm birth. *Long-term tocolysis and hospitalization management was employed for the majority of the patients. Thus, the duration of hospitalization was not included in logistic regression analysis.

TABLE 4: Multivariate analysis of risk factors among SPTBs less than 34 weeks.

\begin{tabular}{lcc}
\hline & Odds ratio (95\% CI) & $P$ value \\
\hline Presurgical CL 0 mm & $5.30(1.58-17.7)$ & 0.007 \\
History of SPTB & $4.65(1.38-15.7)$ & 0.013 \\
Presence of sludge in ultrasound & $4.14(1.20-14.3)$ & 0.025 \\
\hline
\end{tabular}

CL; cervical length, SPTB, spontaneous preterm birth.

preterm singleton livebirth [15]. In addition, two consecutive preterm deliveries increase the risk of subsequent preterm delivery. If a patient has a history of two consecutive very preterm deliveries (SPTB $<30$ weeks), the risk of SPTB is $57 \%$ in the subsequent pregnancy [16]. It is clear that a history of SPTB is one of the most significant risk factors for SPTB based on our results.

An ultrasound-detectable sludge was shown to be a risk for SPTB in this study. A systematic review showed that patients with sludge had higher incidences of SPTB $[15,17]$. Recent studies also showed that amniotic fluid sludge is an independent risk factor for PTB [18-20]. Nulliparous patients with $\mathrm{CL}<30 \mathrm{~mm}$ with sludge between 16 and 22 weeks showed an increase in not only PTB $<32$ weeks (aOR: 2.78 (95\% CI: $1.42-5.45)$ ) but also PTB <34 weeks (aOR: 1.85 (95\% CI: 1.00-3.44)), revealing that the association between sludge and PTB was greater in earlier gestational weeks [19]. 
The sludge is considered to reflect a severe intra-amniotic infection-related inflammatory process [21]. The concentration of a proinflammatory cytokine (interleukin-8) in amniotic fluid and presence of histological chorioamnionitis in patients with sludge significantly increased [22]. It is still unknown whether antibiotics decrease PTB in patients with sludge. A recent study showed that antibiotics decreased not only SPTB $<34$ weeks in patients with shortened CL $(<25 \mathrm{~mm})$ but also the incidence of sludge [23]. Inconsistently, antibiotics (azithromycin or moxifloxacin) for patients with sludge did not decrease PTB $<37$ weeks and $<28$ weeks compared with those without the antibiotics [24]. Patients with sludge are likely to have significant intrauterine infection, and, thus, it may be difficult for emergent cerclage to show effectiveness.

Unmeasurable CL (CL $0 \mathrm{~mm}$ ) was also a risk factor for SPTB in this study. Shortened CL is a well-known risk factor for SPTB, and emergent cerclage shows the preventive effect for SPTB in patients with shortened CL. Berghella et al. [25] showed that patients with $\mathrm{CL}<10 \mathrm{~mm}$, compared with those $>10 \mathrm{~mm}$, were less likely to suffer SPTB $<35$ weeks $(30 / 76$ (40\%) vs. 29/50 (58\%) (RR 0.68 (0.47-0.98)), suggesting that $\mathrm{CL}<10 \mathrm{~mm}$ should be an indication for emergent cerclage in patients even without a history of SPTB. However, emergent cerclage for patients with $\mathrm{CL}<5 \mathrm{~mm}$ did not show the preventive effect significantly (RR 0.79 (0.5-1.23)) [25]. Patients with very short CL such as requiring the replacement of the bag at surgery may have another pathological condition and, thus, emergent cerclage may not show the preventive effect simply. Although it is still unclear how much the cerclage reduces SPTB, unmeasurable CL should be taken into consideration as a high-risk following the emergent cerclage.

Comparing the effectiveness of cerclage or any other treatment with the endpoint being the incidence often causes some difficulty. Our protocol for cerclage may not be completely consistent with the global standard regarding the following two points: (1) Progesterone was not administered, and (2) cerclage was performed for a patient with CL $<25 \mathrm{~mm}$ even without a history of SPTB. A study involving a large number of showed that cerclage decreased the SPTB incidence for a singleton pregnancy in the presence of a history of SPTB with CL $<25 \mathrm{~mm}$, but only in those with a history of SPTB [26]. It remains unknown whether cerclage decreases PTB in patients with $\mathrm{CL}<25 \mathrm{~mm}$ even without a history of SPTB, although several small studies existed. Althuisius et al. reported that emergent cerclage significantly decreased SPTB $<34$ weeks ( $1 / 10$ vs. $5 / 8$ patients; $P=0.04$ ) in a randomized study [27]. On the contrary, a Japanese study also reported that emergent cerclage for patients with $\leq \mathrm{CL} 25 \mathrm{~mm}$ including those without a history of SPTB $(n=106)$ did not decrease SPTB without progesterone administration [28]. A few authors also showed no improvement of perinatal outcomes, including the rate of PTB $[25,29,30]$. Regarding vaginal progesterone administration, a meta-analysis revealed that vaginal progesterone decreased SPTB $<33$ weeks (14\%, RR 0.62, 95\% CI, 0.47-0.81) [31]. As described, we did not use progesterone. This may have prompted us to widen the indication of cerclage: we performed cerclage irrespective of the presence/absence of a history of SPTB. In this study, the rate of SPTB $<34$ weeks after cerclage was $19 \%$. A meta-analysis showed that, of 419 patients with $\mathrm{CL}<25 \mathrm{~mm}$ without a history of SPTB, the incidence of PTB $<34$ weeks was $20.1 \%(45 / 224)$ [30]. This rate is comparable to that $(16 \%)$ in the present study. Considering that the present study included 30 patients with a history of SPTB (considered high-risk), the present results may be satisfactory.

This study had some limitations. First, this was a retrospective analysis, and the study number was relatively small. Second, confounding risk factors could not be completely excluded. For example, intra-amniotic infection can cause SPTB; however, we only checked for the presence or absence of sludge, serum CRP, and cervical elastase. Although interleukin- 6 and -8 in amniotic fluid can indicate intra-amniotic infection [32], we did not evaluate them. Third, as described, the management of threatened PTB is not always consistent with the global standard. As stated, we employed long-term tocolysis with ritodrine hydrochloride and/or magnesium sulfate, and systemic antibiotic administration without progesterone. The management background should be taken into account.

\section{Conclusion}

Three risk factors predicted SPTB $<34$ weeks after emergency cerclage: unmeasurable CL (CL $0 \mathrm{~mm}$ ), a history of SPTB, and the presence of sludge on ultrasound. SPTB $<34$ weeks occurred after emergency cerclage in $16 \%$ of patients, being comparable with the recent data. This study may have indicated the data of cerclage effectiveness without progesterone, data excluding the effect of progesterone.

\section{Data Availability}

The data used to support the findings of this study are available from the corresponding author upon request.

\section{Ethical Approval}

Patient anonymity was preserved, and institutional approval was obtained in our center.

\section{Conflicts of Interest}

The authors declare that they have no conflicts of interest regarding this study.

\section{References}

[1] A. Jarde, O. Lutsiv, J. Beyene, and S. McDonald, "Vaginal progesterone, oral progesterone, 17-OHPC, cerclage, and pessary for preventing preterm birth in at-risk singleton pregnancies: an updated systematic review and network metaanalysis," BJOG: An International Journal of Obstetrics \& Gynaecology, vol. 126, no. 5, pp. 556-567, 2019.

[2] D. M. Haas, T. F. Imperiale, P. R. Kirkpatrick, R. W. Klein, T. W. Zollinger, and A. M. Golichowski, "Tocolytic therapy," Obstetrics \& Gynecology, vol. 113, no. 3, pp. 585-594, 2009. 
[3] K. J. Oh, R. Romero, J. Y. Park et al., "Evidence that antibiotic administration is effective in the treatment of a subset of patients with intra-amniotic infection/inflammation presenting with cervical insufficiency," American Journal of Obstetrics and Gynecology, vol. 221, p. 140, 2019.

[4] N. Medley, J. P. Vogel, A. Care, and Z. Alfirevic, "Interventions during pregnancy to prevent preterm birth: an overview of Cochrane systematic reviews," Cochrane Database of Systematic Reviews, vol. 11, Article ID CD012505, 2018.

[5] A. Premkumar, N. Sinha, E. S. Miller, and A. M. Peaceman, "Perioperative use of cefazolin and indomethacin for physical examination-indicated cerclages to improve gestational latency," Obstetrics \& Gynecology, vol. 135, no. 6, pp. 1409-1416, 2020.

[6] V. Jain, S. D. McDonald, W. R. Mundle, and D. Farine, "Guideline No. 398: progesterone for prevention of spontaneous preterm birth," Journal of Obstetrics and Gynaecology Canada, vol. 42, no. 6, pp. 806-812, 2020.

[7] N. Medley, B. Poljak, S. Mammarella, and Z. Alfirevic, "Clinical guidelines for prevention and management of preterm birth: a systematic review," BJOG: An International Journal of Obstetrics \& Gynaecology, vol. 125, no. 11, pp. 1361-1369, 2018.

[8] Committee on Practice Bulletins-Obstetrics, “The American College of Obstetricians and Gynecologists. Practice bulletin no. 130: prediction and prevention of preterm birth," $\mathrm{Ob}$ stetrics \& Gynecology, vol. 120, pp. 964-973, 2012.

[9] H. Minakami, Y. Hiramatsu, M. Koresawa et al., "Guidelines for obstetrical practice in Japan: Japan society of obstetrics and gynecology (JSOG) and Japan association of obstetricians and gynecologists (JAOG) 2011 edition," Journal of Obstetrics and Gynaecology Research, vol. 37, no. 9, pp. 1174-1197, 2011.

[10] H. Minakami, T. Maeda, T. Fujii et al., "Guidelines for obstetrical practice in Japan: Japan society of obstetrics and gynecology (JSOG) and Japan association of obstetricians and gynecologists (JAOG) 2014 edition," Journal of Obstetrics and Gynaecology Research, vol. 40, no. 6, pp. 1469-1499, 2014.

[11] R. Kawaguchi, K. Matsumoto, S. Akira et al., "Guidelines for office gynecology in Japan: Japan society of obstetrics and gynecology (JSOG) and Japan association of obstetricians and gynecologists (JAOG) 2017 edition," Journal of Obstetrics and Gynaecology Research, vol. 45, no. 4, pp. 766-786, 2019.

[12] Y. Hatakeyama, H. Miura, A. Sato et al., "Neutrophil elastase in amniotic fluid as a predictor of preterm birth after emergent cervical cerclage," Acta Obstetricia et Gynecologica Scandinavica, vol. 95, no. 10, pp. 1136-1142, 2016.

[13] F. Fuchs, M.-V. Senat, H. Fernandez, A. Gervaise, R. Frydman, and J. Bouyer, "Predictive score for early preterm birth in decisions about emergency cervical cerclage in singleton pregnancies," Acta Obstetricia et Gynecologica Scandinavica, vol. 91, no. 6, pp. 744-749, 2012.

[14] A. O. Odibo, C. Farrell, G. A. Macones, and V. Berghella, "Development of a scoring system for predicting the risk of preterm birth in women receiving cervical cerclage," Journal of Perinatology, vol. 23, no. 8, pp. 664-667, 2003.

[15] C. Phillips, Z. Velji, C. Hanly, and A. Metcalfe, "Risk of recurrent spontaneous preterm birth: a systematic review and meta-analysis," BMJ Open, vol. 7, no. 6, Article ID e015402, 2017.

[16] J. McManemy, E. Cooke, E. Amon, and T. Leet, "Recurrence risk for preterm delivery," American Journal of Obstetrics and Gynecology, vol. 196, no. 576, pp. e1-7, 2007.

[17] V. Pergialiotis, I. Bellos, A. Antsaklis, D. Loutradis, and G. Daskalakis, "Presence of amniotic fluid sludge and pregnancy outcomes: a systematic review," Acta Obstetricia et Gynecologica Scandinavica, vol. 99, no. 11, pp. 1434-1443, 2020.

[18] Y. Tsunoda, T. Fukami, K. Yoneyama, I. Kawabata, and T. Takeshita, "The presence of amniotic fluid sludge in pregnant women with a short cervix: an independent risk of preterm delivery," Journal of Maternal-Fetal and Neonatal Medicine, vol. 33, no. 6, pp. 920-923, 2020.

[19] G. R. Saade, E. A. Thom, W. A. Grobman et al., "Eunice kennedy shriver national institute of child health and human development maternal-fetal medicine units (MFMU) network. Cervical funneling or intra-amniotic debris and preterm birth in nulliparous women with midtrimester cervical length less than $30 \mathrm{~mm}$," Ultrasound in Obstetrics and Gynecology, vol. 52, pp. 757-762, 2018.

[20] I. Adanir, O. Ozyuncu, A. F. Gokmen Karasu, and L. S. Onderoglu, "Amniotic fluid "sludge"; prevalence and clinical significance of it in asymptomatic patients at high risk for spontaneous preterm delivery," Journal of Maternal-Fetal and Neonatal Medicine, vol. 31, no. 2, pp. 135-140, 2018.

[21] R. Romero, J. P. Kusanovic, J. Espinoza et al., "What is amniotic fluid'sludge'?" Ultrasound in Obstetrics and Gynecology, vol. 30, no. 5, pp. 793-798, 2007.

[22] N. Yoneda, S. Yoneda, H. Niimi et al., "Sludge reflects intraamniotic inflammation with or without microorganisms," American Journal of Reproductive Immunology, vol. 79, no. 2, 2018.

[23] A. R. Hatanaka, M. S. Franca, T. E. N. K. Hamamoto, L. C. Rolo, R. Mattar, and A. F. Moron, "Antibiotic treatment for patients with amniotic fluid "sludge" to prevent spontaneous preterm birth: a historically controlled observational study," Acta Obstetricia et Gynecologica Scandinavica, vol. 98, no. 9, pp. 1157-1163, 2019.

[24] R. D. Cuff, E. Carter, R. Taam et al., "Effect of antibiotic treatment of amniotic fluid sludge," American Journal of Obstetrics \& Gynecology MFM, vol. 2, no. 1, Article ID 100073, 2020.

[25] V. Berghella, A. Ciardulli, O. A. Rust et al., "Cerclage for sonographic short cervix in singleton gestations without prior spontaneous preterm birth: systematic review and metaanalysis of randomized controlled trials using individual patient-level data," Ultrasound in Obstetrics \& Gynecology, vol. 50, no. 5, pp. 569-577, 2017.

[26] V. Berghella and A. D. Mackeen, "Cervical length screening with ultrasound-indicated cerclage compared with historyindicated cerclage for prevention of preterm birth," Obstetrics \& Gynecology, vol. 118, no. 1, pp. 148-155, 2011.

[27] S. M. Althuisius, G. A. Dekker, H. P. van Geijn, D. J. Bekedam, and P. Hummel, "Cervical incompetence prevention randomized cerclage trial (CIPRACT): study design and preliminary results," American Journal of Obstetrics and Gynecology, vol. 183, no. 4, pp. 823-829, 2000.

[28] K. Otsuki, A. Nakai, Y. Matsuda et al., "Randomized trial of ultrasound-indicated cerclage in singleton women without lower genital tract inflammation," Journal of Obstetrics and Gynaecology Research, vol. 42, no. 2, pp. 148-157, 2016.

[29] O. A. Rust, R. O. Atlas, K. J. Jones, B. N. Benham, and J. Balducci, "A randomized trial of cerclage versus no cerclage among patients with ultrasonographically detected secondtrimester preterm dilatation of the internal os," American Journal of Obstetrics and Gynecology, vol. 183, no. 4, pp. 830-835, 2000.

[30] M. S. To, Z. Alfirevic, V. C. Heath et al., "Cervical cerclage for prevention of preterm delivery in woman with short cervix: 
randomised controlled trial," The Lancet, vol. 363, no. 9424, pp. 1849-1853, 2004.

[31] R. Romero, A. Conde-Agudelo, E. Da Fonseca et al., "Vaginal progesterone for preventing preterm birth and adverse perinatal outcomes in singleton gestations with a short cervix: a meta-analysis of individual patient data," American Journal of Obstetrics and Gynecology, vol. 218, no. 2, pp. 161-180, 2018.

[32] R.-M. Holst, B. Jacobsson, H. Hagberg, and U.-B. Wennerholm, "Cervical length in women in preterm labor with intact membranes: relationship to intra-amniotic inflammation/microbial invasion, cervical inflammation and preterm delivery," Ultrasound in Obstetrics and Gynecology, vol. 28 , no. 6, pp. 768-774, 2006. 